\title{
Recent developments for multi-leg QCD amplitudes with massive particles
}

\section{Rutger Boels}

Niels Bohr Institute, Niels Bohr International Academy

Blegdamsvej 17, DK-2100 Copenhagen, Denmark

and

The Mathematical Institute, University of Oxford

24-29 St. Giles, Oxford OX1 3LP, United Kingdom

\section{Christian Schwinn* ${ }^{\dagger}$}

Institut für Theoretische Physik E

RWTH Aachen, D - 52056 Aachen, Germany

E-mail: schwinnephysik.rwth-aachen. de

\section{Stefan Weinzierl}

Institut für Physik, Universität Mainz.

D - 55099 Mainz, Germany

We review the extension of modern techniques for the calculation of helicity amplitudes in QCD to massive particles. The focus is on the use of supersymmetric Ward identites that relate amplitudes with massive quarks to those with massive scalars, the application of on-shell recursion relations to amplitudes with massive quarks and an extension of the CSW rules to massive scalars.

8th International Symposium on Radiative Corrections

October 1-5, 2007

Florence, Italy

\footnotetext{
*Speaker.

†Supported by the DFG Sonderforschungsbereich/Transregio 9 "Computergestützte Theoretische Teilchenphysik"
} 


\section{Introduction}

Witten's observations on twistor-space properties of QCD scattering amplitudes [1] led to a number of new calculational methods like the diagrammatic rules of Cachazo, Svrčeck and Witten (CSW) [2], the on-shell recursion relations of Britto, Cachazo, Feng and Witten (BCWF) [3, 4] and a renewed interest in unitarity methods for loop calculations [5]. Several challenges remain to turn these methods into viable alternatives to traditional approaches e.g. automatization, the numerical stability and the extension to massive particles. This contribution reviews progress in the latter area. In section 2 Supersymmetry (SUSY) is used to relate the simplest helicity amplitudes of massive quarks and scalars. Extensions of the BCFW and CSW rules to massive quarks and scalars are presented in sections 3 and 4.

\section{MHV amplitudes and SUSY Ward-Identities for massive quarks and scalars}

The simplest helicity amplitudes of gluons are the "maximally helicity violating" (MHV) amplitudes with two negative helicity gluons and an arbitrary number of positive helicity gluons [6]. In spinor-helicity notation the color-ordered partial amplitudes (see e.g. [5]) read

$$
A_{n}\left(g_{1}^{+}, \ldots, g_{i}^{-}, \ldots, g_{j}^{-}, \ldots g_{n}^{+}\right)=\mathrm{i} 2^{n / 2-1} \frac{\langle i j\rangle^{4}}{\langle 12\rangle\langle 23\rangle \ldots\langle(n-1) n\rangle\langle n 1\rangle} .
$$

Color-stripped tree amplitudes of massless quarks in QCD are identical to those of gluinos $\Lambda$ in an unbroken SUSY Yang-Mills theory. Using the fact that the SUSY charge annihilates the vacuum, one can derive SUSY Ward-identities (SWIs) in an unbroken SUSY Yang-Mills theory:

$$
0=\left\langle 0\left|\left[Q_{\mathrm{SUSY}}(\eta), \Phi_{1} \ldots \Phi_{n}\right]\right| 0\right\rangle=\sum_{i}\left\langle 0\left|\Phi_{1} \ldots \delta_{\eta} \Phi_{i} \ldots \Phi_{n}\right| 0\right\rangle .
$$

The SUSY transformations of the helicity states parameterized by an anti-commuting spinor $\eta$ read

$$
\delta_{\eta} g^{ \pm}=\Gamma_{\eta}^{ \pm}(k) \Lambda^{ \pm}, \quad \delta_{\eta} \Lambda^{ \pm}=\Gamma_{\eta}^{\mp}(k) g^{ \pm}
$$

where $\Gamma_{\eta}^{ \pm}(k)=\sqrt{2}\langle\eta \pm \mid k \mp\rangle$. These transformations can be used in the SWI to relate the MHV amplitudes (2.1) to amplitudes with massless quarks [7]:

$$
\langle 1 j\rangle A_{n}\left(\bar{\Lambda}_{1}^{-}, g_{2}^{+}, \ldots, g_{j}^{-}, \ldots, \Lambda_{n}^{+}\right)=\langle n j\rangle A_{n}\left(g_{1}^{-}, g_{2}^{+}, \ldots, g_{j}^{-}, \ldots, g_{n}^{+}\right) .
$$

The MHV amplitudes can serve as building blocks for all massless QCD amplitudes, either as vertices in CSW diagrams [2] or as input in BCFW relations [3, 4]. As a starting point for the extension of such methods one can therefore consider the simplest amplitudes with massive particles, e.g. amplitudes with a pair of massive scalars and positive helicity gluons [8]

$$
A\left(\bar{\phi}_{1}, g_{2}^{+}, \ldots, \phi_{n}\right)=\mathrm{i} 2^{n / 2-1} m^{2} \frac{\left\langle 2+\left|\prod_{j=3}^{n-2}\left(y_{1, j}-\not k_{j} k_{1, j}\right)\right| n-1\right\rangle}{y_{1,2} y_{1,3} \ldots y_{1, n-2}\langle 23\rangle\langle 34\rangle \ldots\langle(n-2)(n-1)\rangle}
$$

where $k_{i, j}=k_{i}+\ldots k_{j}$ and $y_{1, j}=k_{1, j}^{2}-m^{2}$. The amplitudes (2.5) can be related to amplitudes with massive quarks using SWIs in a SUSY Yang-Mills theory with a massive quark $Q$ and two complex massive scalars $\phi^{ \pm}$as super-partners [9]. External states of massive quarks can be introduced as

$$
u( \pm)=\frac{1}{\left\langle p^{b} \mp \mid q \pm\right\rangle}(\not p+m)|q \pm\rangle, \quad \bar{u}( \pm)=\frac{1}{\left\langle q \mp \mid p^{b} \pm\right\rangle}\langle q \mp|(\not p+m)
$$


with the light-like vector $p^{b}=p-p^{2} /(2 p \cdot q) q$ and where $q$ defines the axis of the quark spin. The "helicity" states of the quarks defined by the spinors (2.6) are related to the scalars by SUSY transformations

$$
\delta_{\eta} Q^{ \pm}=-\Gamma_{\eta}^{ \pm}(k) \phi^{ \pm}+\Sigma_{\eta}^{\mp}(k, q) \phi^{\mp}, \quad \delta_{\eta} \phi^{ \pm}=-\Gamma_{\eta}^{\mp}(k) Q^{ \pm}-\Sigma_{\eta}^{\mp}(k, q) Q^{\mp}
$$

where $\Sigma_{\eta}^{ \pm}(k, q)=\sqrt{2} m\langle q \pm \mid \eta \mp\rangle /\langle q \pm \mid k \mp\rangle$. For the choice $|\eta+\rangle \propto|q+\rangle$ the terms proportional to the mass drop out and the transformations become similar to the massless case (2.3). The amplitudes with massive quarks and positive helicity gluons are related to the scalar amplitudes (2.5) by a simple SWI:

$$
\langle 1 q\rangle A\left(\bar{Q}_{1}^{+}, g_{2}^{+}, \ldots, Q_{n}^{-}\right)=\langle n q\rangle A\left(\bar{\phi}_{1}^{+}, g_{2}^{+}, \ldots, \phi_{n}^{-}\right) .
$$

The amplitudes with one negative helicity gluon can also be related to scalar amplitudes [9].

\section{On-shell recursion relations for massive quarks}

The on-shell recursion relations $[3,4,10]$ express tree amplitudes in terms of products of two amplitudes with fewer external particles:

$$
A_{n}\left(\Phi_{1}, \ldots, \Phi_{n}\right)=\sum_{P(i, j), \sigma}^{n} A\left(\Phi_{r}, \ldots, \Phi_{i}^{\prime}, \ldots, \Phi_{s},-\Phi_{K}^{\prime}{ }^{\sigma}\right) \frac{\mathrm{i}}{K^{2}-m^{2}} A\left(\Phi_{K}^{\prime}{ }^{-\sigma}, \ldots, \Phi_{j}^{\prime}, \ldots, \Phi_{r-1}\right) .
$$

The sum is over helicities $\sigma$ and all partitions of momenta $P(i, j)$ into two sets so that $k_{i}$ is in one of the sets and $k_{j}$ in the other one. On the right hand side the two external momenta $k_{i}$ and $k_{j}$ are shifted into the complex plane. For light-like $k_{i}$ and $k_{j}$ the shift is performed by shifting the spinors according to

$$
\left|i^{\prime}+\right\rangle=|i+\rangle-z|j+\rangle, \quad\left|j^{\prime}-\right\rangle=|j-\rangle+z|i-\rangle .
$$

In each term of the relation (3.1), the value of $z$ is chosen so that the internal momentum $K^{\prime}=$ $k_{r}+\cdots+k_{i}^{\prime}+\cdots+k_{s}$ is on-shell.

One approach for the application of on-shell recursion relations to amplitudes of massive particles with spin [11] expresses (3.1) in terms of "stripped" amplitudes with removed external polarization spinors. Recently all amplitudes with a pair of massive quarks and up to four gluons have been calculated in this framework [12]. As shown in [13] one can also treat internal quarks in the helicity formalism which allows to shift also massive quark lines. This can be used to show that all QCD amplitudes (i.e. also those with only massive quarks) can be obtained from BCFW relations and simplifies the structure of the recursion relations in some cases. The shift of two massive momenta $p_{i / j}$ is implemented by decomposing them into two two light-like vectors $l_{i / j}$

$$
p_{i}=l_{i}+\alpha_{j} l_{j}, \quad p_{j}=\alpha_{i} l_{i}+l_{j}
$$

For explicit expressions see [13]. The shift of massive-quark spinors $u_{i}(-)$ and $\bar{u}_{j}(+)$ is defined as

$$
u_{i}^{\prime}(-)=u_{i}(-)-z\left|l_{j}+\right\rangle, \quad \bar{u}_{j}^{\prime}(+)=\bar{u}_{j}(+)+z\left\langle l_{i}+\right| .
$$

Here $\left|l_{j} \pm\right\rangle$ have to be used as reference spinors for a massive quark $Q_{i}$ (and vice versa for $Q_{j}$ ) in order to obtain completeness relations and to avoid spurious poles in $z$ [13]. For an internal massive quark $Q_{K}$ in (3.1), one uses the reference spinors $\left|l_{j}+\right\rangle$ and $\left\langle l_{i}+\right|$. 
The proof of the BCFW relations in [4] continues the scattering amplitude to arbitrary complex values of $z$ and requires $\lim _{z \rightarrow \infty} A(z)=0$. This constrains the helicity of the particles $i$ and $j$. By estimating the $z \rightarrow \infty$ behavior of individual Feynman diagrams as in [4] it follows that the case $\left(i^{+}, j^{-}\right)$is always allowed unless $i$ and $j$ are quarks joined by a fermion line [14]. Using a supplementary three particle shift $[10,13]$ one can show that also the combinations $\left(g_{i}^{+}, g_{j}^{+}\right)$, $\left(g_{i}^{+}, Q_{j}^{+}\right)$and $\left(q_{i}^{+}, Q_{j}^{+}\right)$for a massless quark $q_{i}$ are allowed. Amplitudes with only massive quarks can be obtained by shifting three external legs. The case $\left(i^{-}, j^{-}\right)$is analogous [13].

\section{CSW diagrams for massive scalars}

In the CSW rules [2] QCD amplitudes are constructed from diagrams with vertices that are given by off-shell continuations of the MHV amplitudes (2.1). External massive gauge- and Higgs bosons have been included in this approach in $[15,16]$. In [17] it is shown how to extend the CSW rules to propagating massive scalars using a canonical transformation method [18] and by the construction of a twistor action that reduces to the CSW-Lagrangian in a certain gauge [19]. It is expected that similar rules for massive particles with spin can be derived in an analogous way.

In the approach of [18], one works with the light-cone gauge Lagrangian that contains only the physical modes of the gluon, $A_{z}$ (positive helicity) and $A_{\bar{z}}$ (negative helicity), with interactions of the helicity structure $\mathscr{L}_{++-}^{(3)}, \mathscr{L}_{+--}^{(3)}$ and $\mathscr{L}_{++--}^{(4)}$. A canonical transformation to new variables $B\left[A_{z}\right]$ and $\bar{B}\left[A_{z}, A_{\bar{z}}\right][18]$ can be used to eliminate the non-MHV vertex $\mathscr{L}_{++-}^{(3)}$ in favor of a tower of MHV-vertices $\mathscr{L}_{+\ldots+--}^{(n)}$. A similar procedure can be applied to the light-cone gauge Lagrangian for scalars with the structure

$$
\mathscr{L}^{(2)}(\bar{\phi} \phi)+\mathscr{L}^{(3)}\left(\bar{\phi} A_{z} \phi\right)+\mathscr{L}^{(3)}\left(\bar{\phi} A_{\bar{z}} \phi\right)+\mathscr{L}^{(4)}\left(\bar{\phi} A_{z} A_{\bar{z}} \phi\right)+\mathscr{L}^{(4)}(\bar{\phi} \phi \bar{\phi} \phi)
$$

Using the same transformation $\phi \rightarrow \xi\left[\phi, A_{z}\right]$ for massive and massless scalars in addition to the transformation of the gluons, one can eliminate the non-MHV type coupling $\mathscr{L}^{(3)}\left(\bar{\phi} A_{z} \phi\right)$ and obtains the MHV vertices for massless scalars [20] such as

$$
V_{\mathrm{CSW}}\left(\bar{\xi}_{1}, g_{2}^{+}, \ldots g_{i}^{-}, \ldots \xi_{n}\right)=-\mathrm{i} 2^{n / 2-1} \frac{\langle i n\rangle^{2}\langle 1 i\rangle^{2}}{\langle 12\rangle \ldots\langle(n-1) n\rangle\langle n 1\rangle}
$$

and an additional tower of vertices with a pair of scalars and an arbitrary number of positive helicity gluons that is generated from the transformation of the mass term [17]:

$$
V_{\mathrm{CSW}}\left(\bar{\xi}_{1}, g_{2}^{+}, \ldots \xi_{n}\right)=\mathrm{i} 2^{n / 2-1} \frac{-m^{2}\langle 1 n\rangle}{\langle 12\rangle \ldots\langle(n-1) n\rangle}
$$

As an application, consider the proof of the BCFW recursion relation for a $\left(g_{i}^{+}, g_{j}^{+}\right)$shift. For gluon amplitudes this follows from the CSW representation [4]. The rules given here allow to extend this argument to amplitudes with massive scalars [17], without recourse to auxilary shifts.

\section{Conclusions}

We have reviewed the extensions of new methods for the calculation of scattering amplitudes to massive particles. While we have focused on results for QCD tree amplitudes including massive quarks or scalars, first steps for the extension to the electro-weak theory have appeared $[16,11]$ and some of the on-shell approaches to loop calculations [5] generalize to the massive case. 


\section{References}

[1] E. Witten, Perturbative gauge theory as a string theory in twistor space, Commun. Math. Phys. 252 (2004) 189-258, [hep-th/0312171].

[2] F. Cachazo, P. Svrček, and E. Witten, MHV vertices and tree amplitudes in gauge theory, JHEP 09 (2004) 006, [hep-th/ 0403047$].$

[3] R. Britto, F. Cachazo, and B. Feng, New recursion relations for tree amplitudes of gluons, Nucl. Phys. B715 (2005) 499-522, [hep-th/ 0412308$].$

[4] R. Britto, F. Cachazo, B. Feng, and E. Witten, Direct proof of tree-level recursion relation in Yang-Mills theory, Phys. Rev. Lett. 94 (2005) 181602, [hep-th/ 0501052 ].

[5] Z. Bern, L. J. Dixon, and D. A. Kosower, On-shell methods in perturbative QCD, Annals Phys. 322 (2007) 1587-1634, [arXiv:0704.2798 [hep-ph] ].

[6] S. J. Parke and T. R. Taylor, An amplitude for $n$ gluon scattering, Phys. Rev. Lett. 56 (1986) 2459.

[7] S. J. Parke and T. R. Taylor, Perturbative QCD utilizing extended Supersymmetry, Phys. Lett. B157 (1985) 81

[8] P. Ferrario, G. Rodrigo, and P. Talavera, Compact multigluonic scattering amplitudes with heavy scalars and fermions, Phys. Rev. Lett. 96 (2006) 182001, [hep-th/ 0602043 ].

[9] C. Schwinn and S. Weinzierl, SUSY ward identities for multi-gluon helicity amplitudes with massive quarks, JHEP 03 (2006) 030, [hep-th/ 0602012 ].

[10] S. D. Badger, E. W. N. Glover, V. V. Khoze, and P. Svrček, Recursion relations for gauge theory amplitudes with massive particles, JHEP 07 (2005) 025, [hep-th / 0504159 ].

[11] S. D. Badger, E. W. N. Glover, and V. V. Khoze, Recursion relations for gauge theory amplitudes with massive vector bosons and fermions, JHEP 01 (2006) 066, [hep-th/ 0507161 ].

[12] K. J. Ozeren and W. J. Stirling, Scattering amplitudes with massive fermions using BCFW recursion, Eur. Phys. J. C48 (2006) 159-168, [hep-ph/ 0603071$]$; A. Hall, Massive quark-gluon scattering amplitudes at tree level, arXiv:0710.1300 [hep-ph] .

[13] C. Schwinn and S. Weinzierl, On-shell recursion relations for all born QCD amplitudes, JHEP 04 (2007) 072, [hep-ph/0703021].

[14] M.-x. Luo and C.-k. Wen, Recursion relations for tree amplitudes in super gauge theories, JHEP 03 (2005) 004, [hep-th/0501121].

[15] L. J. Dixon, E. W. N. Glover, and V. V. Khoze, MHV rules for Higgs plus multi-gluon amplitudes, JHEP 12 (2004) 015, [hep-th/ 0411092$].$

[16] Z. Bern, D. Forde, D. A. Kosower, and P. Mastrolia, Twistor-inspired construction of electroweak vector boson currents, Phys. Rev. D72 (2005) 025006, [hep-ph/ 0412167 ].

[17] R. Boels and C. Schwinn, CSW rules for a massive scalar, arXiv:0712.3409 [hep-th].

[18] P. Mansfield, The Lagrangian origin of MHV rules, JHEP 03 (2006) 037, [hep-th/ 0511264 ]; J. H. Ettle and T. R. Morris, Structure of the MHV-rules Lagrangian, JHEP 08 (2006) 003, [hep-th/0605121].

[19] R. Boels, L. Mason, and D. Skinner, From twistor actions to MHV diagrams, Phys. Lett. B648 (2007) 90-96, [hep-th/0702035].

[20] G. Georgiou and V. V. Khoze, Tree amplitudes in gauge theory as scalar MHV diagrams, JHEP 05 (2004) 070, [hep-th/ 0404072$].$ 